\title{
X-Ray Topographic Studies of Defect Structure in $\mathrm{YVO}_{4}$ Crystals
}

K. WieteskA ${ }^{a, *}, \mathrm{~W} . \mathrm{WierzChOWSki}^{b}$, E. WierzBickA ${ }^{b, c}$, A. Malinowska ${ }^{b, d}$, M. Lefeld-SosnowskA ${ }^{c}$, T. LukAsieWicz $^{b}$ AND W. GRAEFF ${ }^{e}$

${ }^{a}$ Institute of Atomic Energy, 05-400 Otwock-Świerk, Poland

${ }^{b}$ Institute of Electronic Materials Technology

Wólczyńska 133, 01-919 Warsaw, Poland

${ }^{c}$ Institute of Experimental Physics, University of Warsaw

Hoża 69, 00-681 Warsaw, Poland

${ }^{d}$ Faculty of Physics, Warsaw University of Technology

Koszykowa 75, 00-662 Warsaw, Poland

${ }^{e}$ HASYLAB at DESY, Notkestr. 85, 22-603 Hamburg, Germany

The perfection of $\mathrm{YVO}_{4}$ crystals, which are predicted to replace formerly used YAG garnets due to higher quantum efficiency and lower excitation level, was studied. The investigations of Czochralski grown undoped $\mathrm{YVO}_{4}$ single crystals were performed mainly by means of X-ray topographic methods. Both synchrotron and conventional X-ray sources were used. The study revealed relatively high density of weak point-like contrasts which can be most probably interpreted as dislocation outcrops. In regions of the crystal close to its boundary we observed glide bands. It was also found that in some regions the dislocations form local subgrain boundaries. The white beam back reflection and monochromatic beam topography allowed to evaluate a local misorientation which not exceeded several angular minutes. No segregation fringes were observed proving a good homogeneity of chemical composition.

PACS numbers: $61.72 . \mathrm{Ff}$

\section{Introduction}

$\mathrm{YVO}_{4}$ is of zirconium type tetragonal structure with space group $I 4_{1} /$ amd and lattice parameters: $a=b=0.712 \mathrm{~nm}$ and $c=0.629 \mathrm{~nm} \mathrm{[1,2].} \mathrm{Yttrium}$ orthovanadate crystals are modern material in laser technology replacing formerly

${ }^{*}$ corresponding author; e-mail: k.wieteska@cyf.gov.pl 
used YAG garnets due to higher quantum efficiency and lower excitation level. Nd-doped $\mathrm{YVO}_{4}$ is an excellent material for making the diode-pumped solid lasers. It is especially useful in microlaser systems excited with semiconductor laser diodes. However, it is very difficult to obtain defect free $\mathrm{YVO}_{4}$ single crystals. Defects, such as dislocations, low-angle boundaries, inclusions can significantly decrease the efficiency of the laser elements. Up to our best knowledge no topographic investigations concerning $\mathrm{YVO}_{4}$ crystals were described in the literature. Some recent results concerning growth and defects of $\mathrm{YVO}_{4}$ are described in $[2-6]$.

In the present work a number of samples cut out from a Czochralski grown non-doped $\mathrm{YVO}_{4}$ crystals were studied with several X-ray topographic methods exploring both synchrotron and conventional X-ray sources. The synchrotron investigations were performed both in white and monochromatic X-ray beam. All topographs were obtained in back reflection geometry.

\section{Samples and experimental procedure}

The $\mathrm{YVO}_{4}$ single crystals were grown by the Czochralski method in the [100] direction, using Cyberstar oxypuller. The compound $\mathrm{YVO}_{4}$ available in form of grade $4.5 \mathrm{~N}$ trade product was used as a charge material. The growth thermal system consisted of iridium crucible (50 mm diameter, $50 \mathrm{~mm}$ high) with passive iridium afterheater (55 $\mathrm{mm}$ diameter, $60 \mathrm{~mm}$ high). The pulling rate was 1-1.2 $\mathrm{mm} / \mathrm{h}$ and rate of growing crystal rotation $6-8 \mathrm{rpm}$. The growth processes were performed in the $\mathrm{N}_{2}$ atmosphere to protect iridium elements and controlled by computer program. The grown crystals were annealed in resistance heater in $1100^{\circ} \mathrm{C}$ during $5 \mathrm{~h}$ in air atmosphere and then cooled slowly during $25 \mathrm{~h}$. The crystals were of rectangular cross-section about $20 \times 22 \mathrm{~mm}$ and of $40 \mathrm{~mm}$ length (Fig. 1). They were free from bubbles, twins, and cracks.

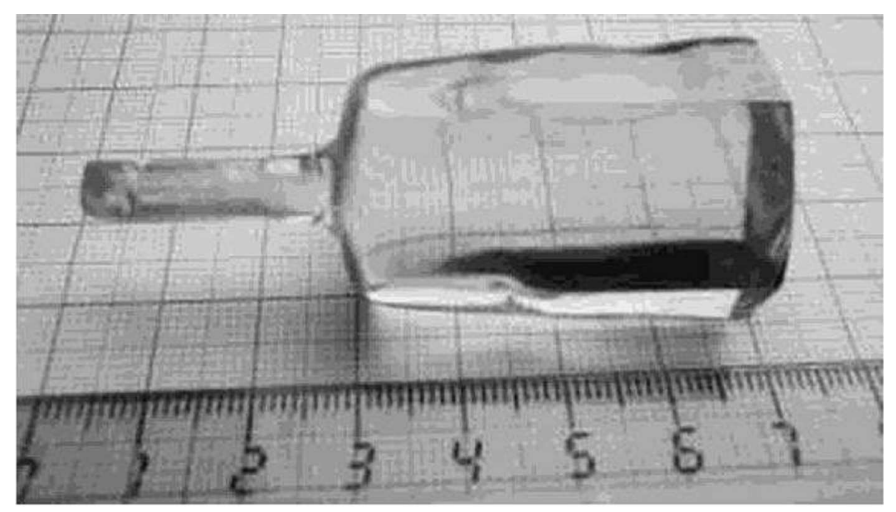

Fig. 1. The investigated undoped $\mathrm{YVO}_{4}$ crystal. 
The samples denoted respectively A, B and C were cut out perpendicular to the growth axis from different regions of the crystal. The sample A was cut out from the region near the seed, $\mathrm{B}$ from the middle, and $\mathrm{C}$ from the end part of the same crystal. All samples were mechanically polished to the thickness of $300 \mu \mathrm{m}$. A set of samples was selected to provide more complete information about the whole crystals. The growth condition in the starting and end parts are usually different than those in the middle part providing the most useful material for applications.

Some different topographic methods exploring both conventional and synchrotron sources of X-rays were used for the characterization of the crystal. An important advantage of conventional topographic methods is the possibility of reproducing the whole area of investigated samples. The presently used synchrotron methods usually reproduce an area with a diameter of several millimeters providing high spatial resolution.

Conventional projection X-ray topographs were taken in back reflection geometry using Mo $K_{\alpha_{1}}$ radiation. The topographs were recorded on $50 \mu \mathrm{m}$ Ilford L4 nuclear plates.

The synchrotron radiation experiments were realized at stations F1 (white beam) and E2 (monochromatic beam) at HASYLAB (DESY, Hamburg) in back reflection geometry. In case of white beam synchrotron projection topographs (SRWB) relatively low glancing angles of $5^{\circ}$ were used. The topographs were recorded on film placed perpendicularly to the incident beam at the distances of 10 and $20 \mathrm{~cm}$ from the crystal.

The samples were also studied by taking monochromatic beam topographs in $0.115 \mathrm{~nm}$ radiation. The beam was formed by successive 333 and 511 symmetrical reflections from silicon crystals. In some cases, due to strong curvature of the sample, the "zebra patterns" were taken. This kind of topograph is obtained, when the crystal position is stepwise altered during exposition by a relatively small angle. In case of deformed crystal the "zebra pattern" reveals a series of stripes corresponding to the regions of crystal reflecting at each subsequent angular setting. In actual case the angular step was equal to $0.003^{\circ}$. The synchrotron topographs were recorded for different azimuths of the crystal either on KODAK SR45 or Slavish holographic films. In all reproduced topographs dark tones correspond to high X-ray intensity.

\section{Results and discussion}

The topographic images of the samples A, B, and C are shown in Figs. 2, 3 , and 4 , respectively. It should be noticed that the topographs obtained with all used methods did not reveal any segregation fringes in all three samples proving high homogeneity of chemical composition. The conventional back reflection topograph and SWBR topographs revealed a relatively high density $\left(<10^{4} \mathrm{~cm}^{-2}\right)$ of weak point-like contrasts, which can be most probably interpreted as dislocation 

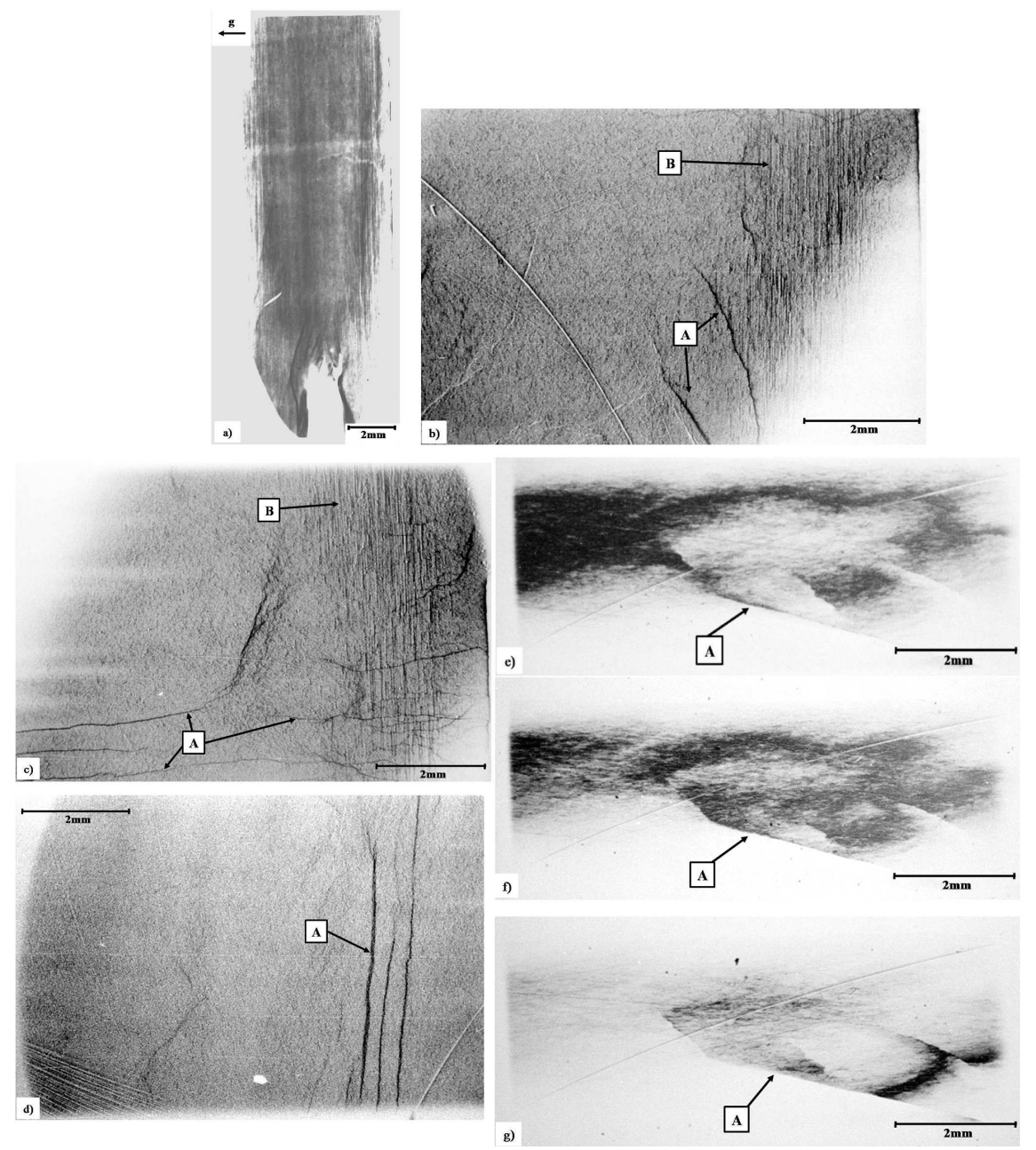

Fig. 2. X-ray projection topographs of sample A cut out near the seed of undoped $\mathrm{YVO}_{4}$ crystal $(d=300 \mu \mathrm{m})$ : (a) back reflection (800) reflection; (b)-(c) SRWB back reflection of two different regions, direction of the beam projection along the normal to (001) planes; (d) SRWB back reflection, direction of the beam projection along the normal to (010) planes; (e)-(g) SR monochromatic plane wave, (400) reflection, $\lambda=$ $0.115 \mathrm{~nm}$, direction of the beam projection along the normal to (001) planes. The pictures were taken for three angular positions passing the maximum of the rocking curve; A - representative subgrain boundaries, B — representative glide bands.

outcrops. In some regions of the crystal close to its boundary we observed some glide bands as it may be seen in Fig. 2b, c. It was also found that in some regions the dislocations form local subgrain boundaries. The representative subgrain boundaries were marked in the topographs by "A" and representative glide bands 


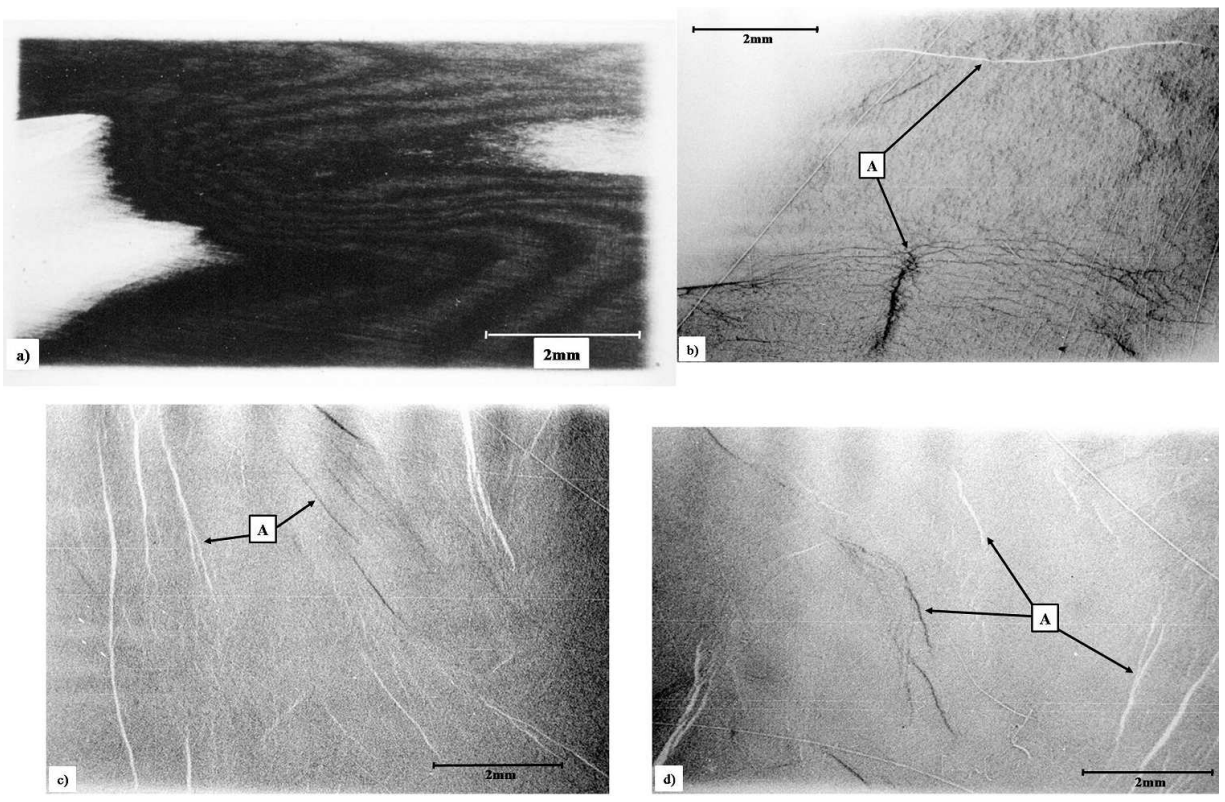

Fig. 3. X-ray projection topographs of sample B $(d=300 \mu \mathrm{m})$ : (a) SR monochromatic wave "zebra" pattern, (400) reflection, $\lambda=0.115 \mathrm{~nm}$, angular step $0.003^{\circ}$, direction of the beam projection along the normal to (001) planes; (b)-(c) SRWB back reflection, direction of the beam projection along the normal to (001) planes, two different places; (d) SRWB back reflection, direction of the beam projection along the normal to (010) planes; A - representative subgrain boundaries.

were marked by "B". Some of the subgrain boundaries form both distinct black or white contrast depending on the sign of mutual misorientation of neighboring grains. One can also notice many smaller subgrain boundaries with less distinct contrast presumably not connected with significant lattice misorientation. The characteristic feature of white beam topographs is a possibility of reproduction of significantly misoriented fragments of the crystal contrary to the conventional back-reflection Lang topography (shown in Fig. 1a), where some boundary parts of the crystal are out of reflection range. The topographs taken with the use of monochromatic beam are very sensitive to local lattice misorientation and enable revealing separate subgrains as may be seen in Fig. 2e-g.

The misorientation of lattice connected with the boundaries was studied in a number of synchrotron monochromatic beam experiment consisting on taking a number of pictures for stepwise changed angular position. This experiment allowed the evaluation of misorientation in the range not exceeding single angular minutes.

The main difference between the three regions of the crystal comes from the subgrain structure which seems to be most extended in the end part (sample C) and relatively poor in the sample from the middle of the crystal. The low number 

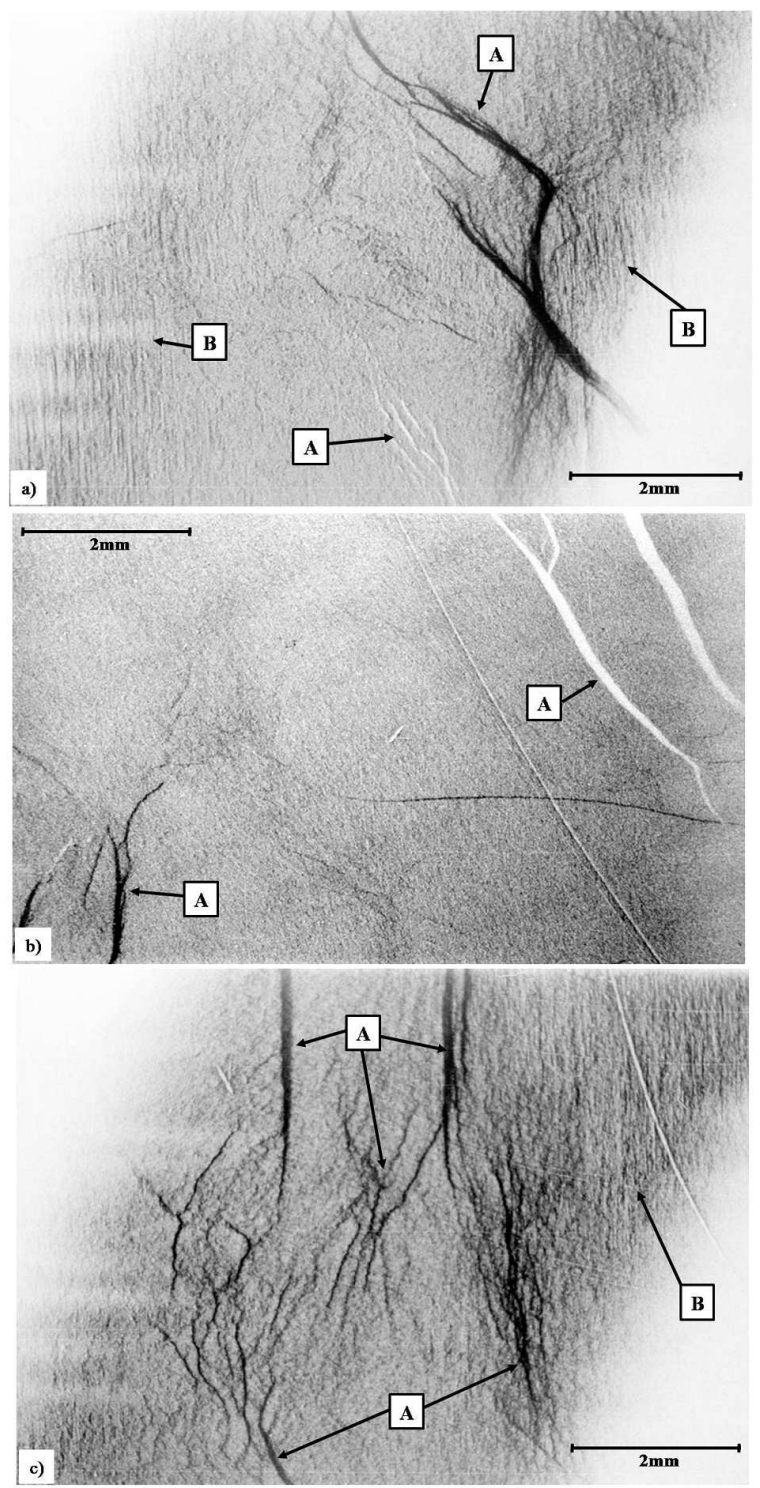

Fig. 4. X-ray projection topographs of sample C $(d=300 \mu \mathrm{m})$ : (a)-(b) SRWB back reflection, direction of the beam projection along the normal to (001) planes, two different places; (c) SRWB back reflection, direction of the beam projection along the normal to (010) planes; A - representative subgrain boundaries, B - representative glide bands.

of subgrain boundaries corresponds, however, to significant lattice bending seen especially in Fig. 3d where relatively large area of the sample had to be reproduced using "zebra" pattern technique. Contrary to that in the sample A with larger number of subgrains the monochromatic beam topographs revealed larger unbent 
blocks with mutual misorientation of several minutes as may be seen in Fig. 2e-g. Extended complex structure of subgrain boundary may be particularly seen in Fig. 4b. Also the width of the grain boundaries contrasts was largest in the case of the sample cut out from the end part of the crystal. The larger number of subgrain boundaries and the extension of their contrast indicate larger stress occurring in the growth process. In case of top part crystal the larger strains are usually connected with enlarging of crystal diameter while in the case of the end part the formation of large strain is obviously connected with stopping of the growth process.

\section{Conclusions}

The $\mathrm{YVO}_{4}$ undoped crystals were grown by the Czochralski method providing crystals of rectangular cross-section about $20 \times 22 \mathrm{~mm}$ and of $40 \mathrm{~mm}$ length free of visible cracks and inclusions.

The conventional and synchrotron X-ray topographic investigations were performed in back reflection geometry revealing the dislocation outcrops, glide bands, and subgrain boundaries. The generation of these defects is usually attributed to the thermal stresses present in the growth process. The mutual misorientation of crystal blocks was evaluated to be of the range of several minutes.

The lowest concentration of subgrains was observed in the sample cut out from the middle part of the crystal, which is usually grown in more stable thermal conditions.

\section{Acknowledgments}

The technical assistance of J. Bondziul is much appreciated.

\section{References}

[1] S. Wu, G. Wang, J. Xie, X. Wu, G. Li, J. Cryst. Growth 249, 176 (2003).

[2] S. Wu, G. Wang, J. Xiea, J. Cryst. Growth 266, 496 (2004).

[3] H. Zhang, Y. Yu, Y. Cheng, J. Liu, H. Li, W. Ge, X. Cheng, X. Xu, J. Wang, M. Jiang, J. Cryst. Growth 283, 438 (2005).

[4] M. Kruczek, E. Talik, H. Sakowska, W. Szyrski, Z. Ujma, D. Skrzypek, J. Cryst. Growth 275, e1715 (2005).

[5] B.Q. Hu, Y.Z. Zhang, X. Wu, X.L. Chen, J. Cryst. Growth 226, 511 (2001).

[6] D.E. Eakins, J.B. LeBret, M.G. Norton, D.F. Bahr, J. Cryst. Growth 266, 411 (2004). 